\title{
Case report of long-term follow-up of guided bone regeneration technique and implant restoration at the mandibular molar area with severe alveolar bone loss from a periapical lesion
}

\author{
Inseok Hong ${ }^{1}$, Jung Ho Park ${ }^{1}$, Hyun Seok ${ }^{1,2}$, Dae-Ho Leem ${ }^{1,2}$ \\ 'Department of Oral and Maxillofacial Surgery, School of Dentistry, Chonbuk National University Dental Hospital, Jeonju, \\ ${ }^{2}$ Research Institute of Clinical Medicine-Biomedical Research Institute, Chonbuk National University Hospital, Jeonju, Korea
}

This report presents a case of long-term observations after guided bone regeneration and implant repair in a patient who showed severe alveolar bone loss from a periapical lesion of \#46. The patient received cyst enucleation and extraction of \#46 under general anesthesia, and underwent guided bone regeneration after 18 months. The implant was placed in the following year, and the patient was followed up for eleven years. (JOURNAL OF DENTAL IMPLANT RESEARCH 2020;39(3):25-28)

Key Words: Dental implant, Guided bone regeneration, Periapical lesion

\section{INTRODUCTION}

Dental implants are the standard when teeth are lost for various reasons. Most periapical lesions are expected to be healed naturally by removing the microbial infection and bacteria ${ }^{1)}$. However, if the tooth gets extracted due to a progressed periapical lesion, bones may not be healed sufficiently for an implant placement. Bone grafts and other techniques are performed to enhance bone regeneration at the lesion ${ }^{2}$. There is a controversy over the necessity of a bone graft ${ }^{3)}$. Also, it is important to consider the timing of bone graft and implant placement after the removal of the lesion. The size of the lesion, the anatomical structure around the lesion, and the other factors must be considered for the best method and timing which remain controversial ${ }^{4)}$.

Remodeling of degenerated bone tissue due to a pathologic condition takes about one to two years ${ }^{3,5}$. If the vertical bone loss is severe even after the remodeling period, a bone graft becomes challenging. For this type of bone graft, it is recommended to use autogenous bone which has a high osteogenetic properties ${ }^{6}$.

This case report is about a patient who received guided bone regeneration with autograft and implant placement after severe alveolar bone loss due to a periapical lesion. The patient was followed for eleven years.

\section{CASE REPORT}

The study was approved by the Chonbuk National University Hospital Institutional Review Board (IRB No. CUH 2020-08-001). In March 2009, a 53-year-old woman was referred to the Department of Oral and Maxillofacial Surgery at Chonbuk National University Hospital for a tooth extraction and the removal of the periapical lesion. From a radiograph, it showed a radiolucent lesion at the apex of \#46 which was close to the inferior alveolar nerve (Fig. 1A).

In April 2009, the extraction of \#46 and cyst enucleation were performed under general anesthesia. There was

Received August 20, 2020, Revised September 4, 2020, Accepted September 4, 2020.

(c) Journal of Dental Implant Research.

(i) This is an open access article distributed under the terms of the Creative Commons Attribution Non-Commercial License (http://creativecommons.org/licenses/by-nc/4.0) which permits unrestricted non-commercial use, distribution, and reproduction in any medium, provided the original work is properly cited.

Correspondence to: Dae-Ho Leem, Department of Oral and Maxillofacial Surgery, School of Dentistry, Chonbuk National University Dental Hospital, 20 Geonji-ro, Deokjin-gu, Jeonju 54907, Korea. Tel: +82-63-250-2068, Fax: +82-63-250-2089, E-mail: idisho@jbnu.ac.kr 

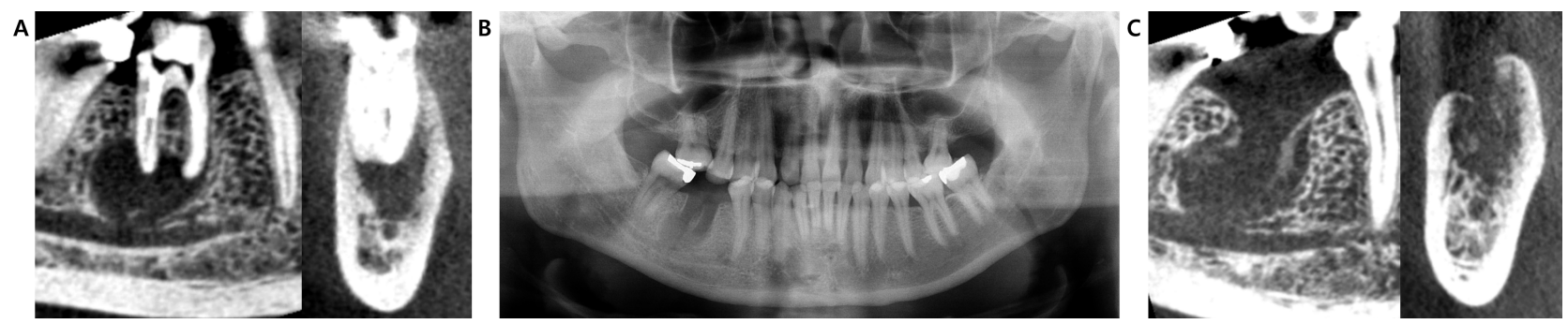

Fig. 1. (A) Computed tomography image of initial visit. (B) Post-op panoramic image. (C) Computed tomography image of post-op after 6 months.
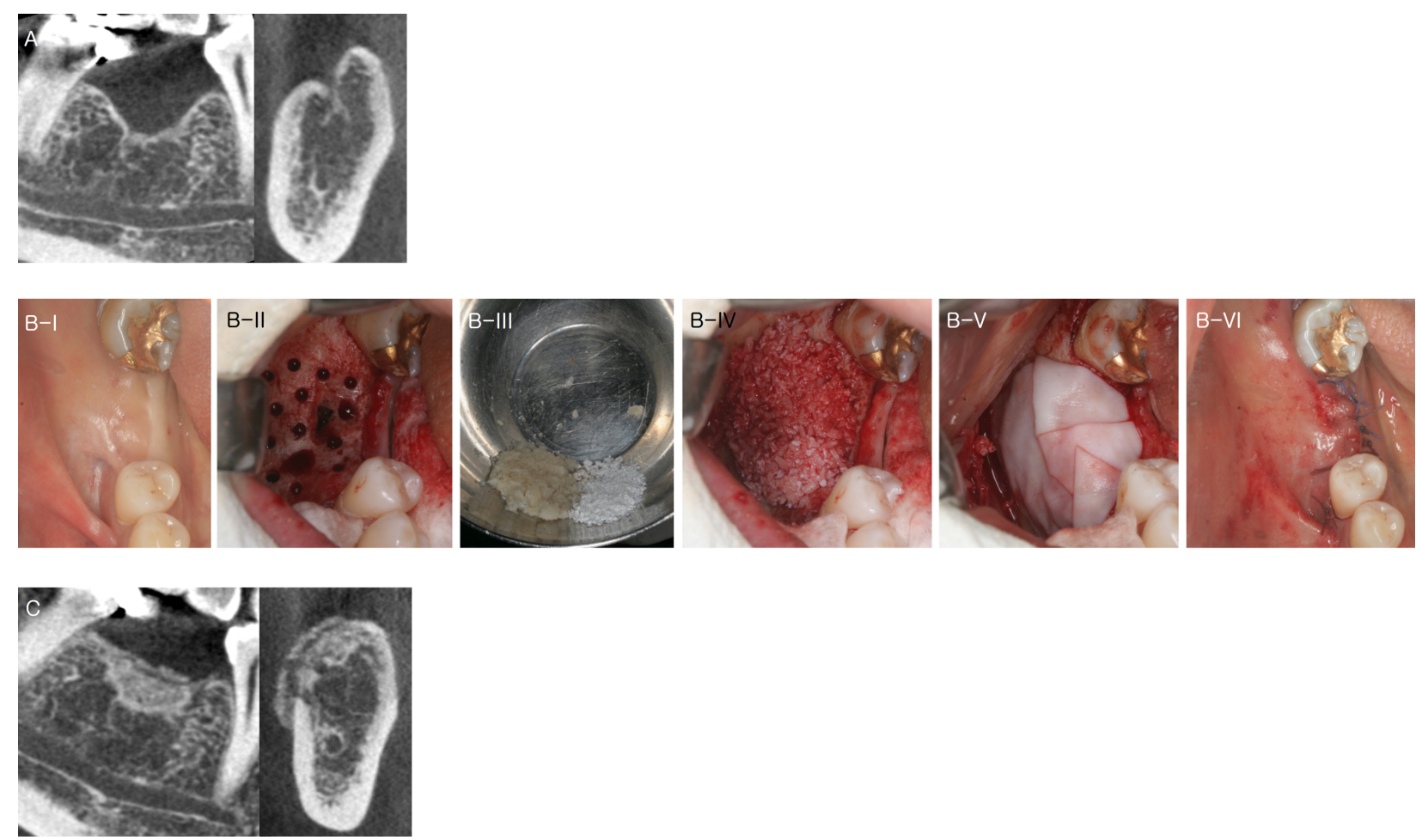

Fig. 2. (A) Computed tomography image of post-op after 18 months. (B) Intra oral view of GBR. (B-I) Pre-op. (B-II) Buccal and occlusal cortical bone perforation. (B-III) Left: Particulated ramus bone, Right: xenogenic bone (Bio-Oss, Geistleich Pharmaceutical, Wolhusen, Switzerland). (B-IV) Mixed particulated bone bulid up. (B-V) The appearance of collagen membrane (BioGide, Geistleich Pharmaceutical, Wolhusen, Switzerland) applied. (B-VI) Sutured state. (C) Computed tomography image of GBR post operatively.

no nerve exposure during the surgery. After the operation, the patient showed a good healing without a complication (Fig. 1B). The biopsy result came out as acute suppurative inflammation and granulation tissue formation.

From the six month postoperative radiograph, the patient showed a good healing, but the bone density at the operated site was low, and the height of the buccal bone was more vertically absorbed than the lingual bone (Fig. 1C).

After the 18 month follow up, guided bone regeneration was decided (Fig. 2A). In January 2011, the guided bone regeneration was performed. About $2 \mathrm{cc}$ of bone was harvested from the right side of ramus using a micro-saw and chisel \& mallet. The collected bone was crushed using a dental bone crusher and mixed with xenogenic bone (0.5 g) (Bio-Oss, Geistleich Pharmaceutical, Wolhusen, Switzerland). The buccal and occlusal cortical bone of \#46 were decorticated, and the mixed bone was grafted, and covered by a collagen membrane (BioGide, Geistleich Pharmaceutical, Wolhusen, Switzerland). Fibrin sealant (Tisseel, Baxter AG, Vienna, Austria) was applied to the operated site, and the site was sutured using subgingival suture, vertical mattress, and inter- 

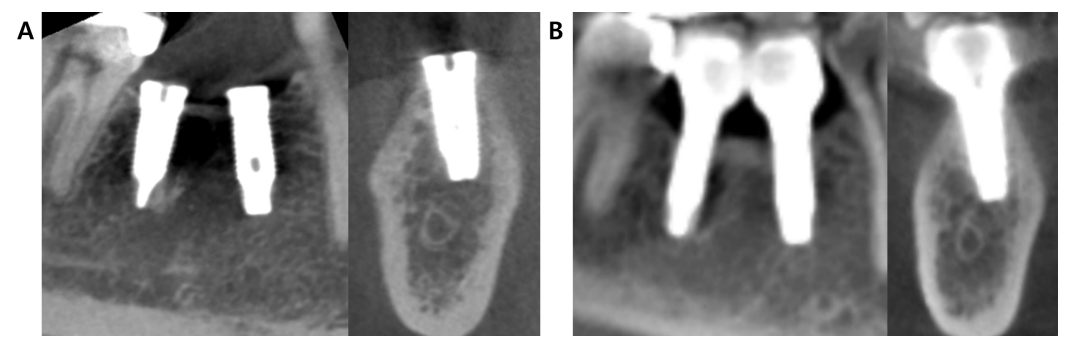

Fig. 3. (A) Computed tomography image of implant placement. (B) Computed tomography image of seven years after the prosthetic loading.

rupted suture technique using 4-0, 5-0 synthetic absorbable sterile suture (Vicryl, Ethicon Incorporation, Somerville, USA) (Fig. 2B). After the GBR, the healing was observed without any complication (Fig. 2C and 3A).

In May 2012, the patient visited the clinic and wanted to place an implant. The patient received the dental implants (Fig. 3B). After the six months, the second surgery was performed, and referred to the prosthodontics department for an implant crown. The patient had the crown restoration and followed for 4 months until the patient was lost for follow up.

In July 2020, the patient visited periodontics department at Chonbuk National University Hospital there was no specific findings from Panorama and Computer tomography (Fig. 3C).

\section{DISCUSSION}

This case is about a patient who underwent tooth extraction and cyst enucleation due to severe periapical lesion at a mandibular molar. The patient was followed for 18 months for bone regeneration and the implants were placed. The patient was followed for eleven years.

There are controversies about whether the bone graft is necessary after a large lesion has been removed from the jaw ${ }^{2,3}$. The period and process of bone regeneration were discussed in various papers, and Di Dio et al. ${ }^{3)}$ reported that all lesions recovered within one year after removal of the cyst without the bone graft. Chiapasco et al. ${ }^{5)}$ observed increased bone density by $48 \%$ at the operated site one year postoperatively, and showed 91\% increased bone density after 2 years without any type of graft. In this case, the conservative treatment was chosen to reduce the risk of infection. At 18 months follow-up, the bone remodeling was completed, but the amount of bone formed was inappropriate.

It is believed that immediate implant placement at the extraction site shortens the entire treatment period and preserves the alveolar walls ${ }^{7}$. However, it was contraindicated to perform immediate implant placement along with bone graft where the tooth showed periapical lesions ${ }^{8,9)}$. However, there are cases of osseointegration in animal experiments even with periapical lesions or periodontal disease $\mathrm{e}^{10)}$. There are similar arguments for humans ${ }^{4,11)}$. Immediate implant placement after tooth extraction with a periapical lesion has the advantage of reducing the burden of the patient about the surgery and the entire treatment period. The downside is that there is a possibility of contaminating the implant due to the remnants of the infection that have not been completely removed $^{11)}$. If the operated site becomes infected and is not properly treated or removed, the bone graft and implant will be failed ${ }^{12)}$. Therefore, several papers consider pre-operative antibiotic administration, pre-operative chlorohexidine gargle, and thorough curettage with irrigation regarding the treatment of apical lesions, ${ }^{4,10,11)}$. Even if the apical lesion has undergone thorough treatment, it is recommended that the immediate implant placement should be delayed if any of the followings are present. (1) Continuous observation of purulent exudates. (2) Soft-tissue cellulitis and granulation tissue at adjacent site. (3) Close to important anatomical structure (Ex. mandibular neurovascular bundle, maxillary sinus, and nasal cavity). (4) Poor anatomical configuration of remaining bone. (5) Any contraindications to treatment, such as systemic diseases (e.g. diabetes). (6) Medication that might compromise healing or osseointegration. (7) Heavy smok$\mathrm{er}^{13)}$. This patient showed insufficient bone shape and amount which the implant placement was postponed for the bone remodeling and to prevent the possibility of 
infection

Lindeboom et al. ${ }^{11)}$ extracted and enucleated a tooth with periapical lesion and placed the implant immediately in 50 patients. During the implant placement, the bone was harvested from the retromolar region and chin, grafted through the hole made at the affected buccal bone plate of the lesion. As a result of one year follow-up, 92\% of them survived. However, there was no mention of the size of the periapical lesion, and the study was limited to an environment with a good bone shape which was enough to produce an initial fixation force of $25 \mathrm{~N}$ upon immediate implant placement. Siegenthaler et al. ${ }^{4)}$ performed immediate implant placement with GBR using xenogenic bone (Bio-Oss, Geistleich Pharmaceutical, Wolhusen, Switzerland) after the extraction of the tooth with periapical lesions for 17 patients. The authors reported that $100 \%$ of the survival rate at the 1 year follow up after GBR.

However, there is nearly no studies showing on the long-term prognosis of implants placed in with these periapical lesions. This report shows a successful result through a long-term follow-up for 11 years, but more cases are needed for generalization.

\section{CONCLUSION}

This report waited for bone regeneration where showed significant bone loss from a severe periapical infection. Guided bone regeneration was postponed until the complete bone remodeling. Guided bone regeneration with autograft and implant placement were performed and followed for eleven years for successful result.

\section{CONFLICT OF INTEREST}

The authors declare that they have no competing interests.

\section{ORCID}

Inseok Hong, https://orcid.org/0000-0003-0114-0534
Hyun Seok, https://orcid.org/0000-0002-5278-3807

\section{REFERENCES}

1. Oztan MD. Endodontic treatment of teeth associated with a large periapical lesion. Int Endod J 2002;35:73-8.

2. Ihan Hren N, Miljavec M. Spontaneous bone healing of the large bone defects in the mandible. Int J Oral Maxillofac Surg 2008;37:1111-6.

3. Di Dio M, Scarapecchia D, Porcelli D, Arcuri C. Spontaneous bone regeneration after removal of cysts: one-year follow-up of 336 consecutive cases. JOURNAL OF ORAL SCIENCE \& REHABILITATION 2016;2:50-6.

4. Siegenthaler DW, Jung RE, Holderegger C, Roos M, Hammerle $\mathrm{CH}$. Replacement of teeth exhibiting periapical pathology by immediate implants: a prospective, controlled clinical trial. Clin Oral Implants Res 2007;18:727-37.

5. Chiapasco M, Rossi A, Motta JJ, Crescentini M. Spontaneous bone regeneration after enucleation of large mandibular cysts: a radiographic computed analysis of 27 consecutive cases. J Oral Maxillofac Surg 2000;58:942-9.

6. Stirling Craig E, Yuhasz M, Shah A, Blumberg J, Salomon J, Lowlicht $R$ et al. Simulated surgery and cutting guides enhance spatial positioning in free fibular mandibular reconstruction. Microsurgery 2015;35:29-33.

7. Lazzara RJ. Immediate implant placement into extraction sites: surgical and restorative advantages. Int J Periodontics Restorative Dent 1989;9:332-43.

8. Tolman DE, Keller EE. Endosseous implant placement immediately following dental extraction and alveoloplasty: preliminary report with 6-year follow-up. Int J Oral Maxillofac Implants 1991;6:24-8.

9. Barzilay I. Immediate implants: their current status. International Journal of Prosthodontics 1993;6:169-9.

10. Novaes Jr AB, Vidigal Jr GM, Novaes AB, Grisi MF, Polloni S, Rosa A. Immediate implants placed into infected sites: a histomorphometric study in dogs. Int J Oral Maxillofac Implants. 1998;13:422-7.

11. Lindeboom JA, Tjiook Y, Kroon FH. Immediate placement of implants in periapical infected sites: a prospective randomized study in 50 patients. Oral Surg Oral Med Oral Pathol Oral Radiol Endod. 2006;101:705-10.

12. Esposito M, Grusovin MG, Kwan S, Worthington HV, Coulthard P. Interventions for replacing missing teeth: bone augmentation techniques for dental implant treatment. Cochrane Database Syst Rev. 2008;3:CD003607.

13. Ebenezer V, Balakrishnan K, Asir RV, Sragunar B. Immediate placement of endosseous implants into the extraction sockets. J Pharm Bioallied Sci. 2015;7:S234-7. 